\title{
Improved Langevin Methods for Spin Systems
}

\author{
I T Drummond and R R Horgan \\ Department of Applied Mathematics and Theoretical Physics \\ University of Cambridge \\ Silver St \\ Cambridge, England CB3 9EW
}

March 21, 2018

\begin{abstract}
We investigate methods for variance reduction and the elimination of systematic error in a Fourier accelerated Langevin scheme for general spin models. We present results for the $S U(3) \times S U(3) / S U(3)$ model in two-dimensions that are consistent with those from multigrid methods. We argue that the timing for the Langevin method makes it comparable to multi-grid for a given level of error.
\end{abstract}

DAMTP-92-72 


\section{Introduction}

Langevin simulation methods may be applied to any continuous spin model [1]. While certain models of the $O(N)$ type may respond best to cluster algorithms [2] there is no comparable method for those of $S U(N)$ or $C P(N)$ type. These have been tackled by multi-grid methods with good results

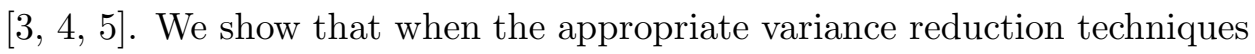
and extrapolation methods for the removal of systematic errors have been applied, the Langevin scheme can compete on a reasonably equal footing with the multi-grid approach at least for the case $N=3$. Higher values of $N$ remain to be investigated. A preliminary version of this work was reported at Lattice91 [6]

\section{Langevin Scheme}

For the purposes of exposition we will assume that we are dealing with an $S U(N) \times S U(N) / S U(N)$ model on an $L^{D}$ hyper-cubical lattice. We will report results with $N=3$ and $D=2$ for various values of $L$. The action is

$$
A(S)=\frac{g}{2} \sum_{n, \mu}\left(\operatorname{Tr}\left(S_{n+\mu}^{\dagger} S_{n}\right)+\text { c.c. }\right)
$$

where $S_{n}$ is an element of $S U(N)$ associated with site $n$ and $\mu$ runs over forward pointing nearest neighbour sites. The probability distribution we wish to simulate is $P_{0}(S) \propto \exp (A(S))$. This distribution is the stationary solution of the Fokker-Planck equation

$$
\frac{\partial}{\partial \tau} P(S, \tau)=H P(S, \tau)
$$

where

$$
H=\sum_{n m a} \kappa_{n m} D_{n}^{a}\left(D_{m}^{a}-U_{m}^{a}\right)
$$

Here $D_{n}^{a}$ is the covariant derivative appropriate to site $n$ in group direction $a$ defined as follows [9]. Let $\left\{\Lambda^{a}\right\}$ be a basis of generators for the defining representation of $S U(N)$. They satisfy the Lie algebra relation $\left[\Lambda^{a}, \Lambda^{b}\right]=$ $C_{a b c} \Lambda^{c}$ where the $\left\{C_{a b c}\right\}$ are the structure constants of the group. Let $e^{\epsilon . \Lambda} S$ stand for the set of spins $\left\{e^{\epsilon_{n}^{a} \Lambda^{a}} S_{n}\right\}$. Then

$$
D_{n}^{a} f(S)=\left.\frac{\partial}{\partial \epsilon_{n}^{a}} f\left(e^{\epsilon . \Lambda} S\right)\right|_{\epsilon=0} .
$$


The covariant derivatives satisfy the Lie algebra relation $\left[D_{n}^{a}, D_{m}^{b}\right]=-\delta_{n m} C_{a b c} D_{n}^{c}$. The drift term in the Fokker-Planck equation is $U_{n}^{a}=D_{n}^{a} A(S)$. Clearly for any choice of the "diffusivity" tensor $\kappa_{n m}$ we have $H P_{0}(S)=0$.

The presence of $\kappa_{n m}$ allows us to exploit the idea of Fourier acceleration [10]. In order to preserve translation invariance $\kappa_{n m}$ depends only on the separation $n-m$ of the two sites. The precise form of the its functional depencence is fixed by specifying the shape of its Fourier transform. Guided by ideas of free field theory we choose 10

$$
\tilde{\kappa}(k)=\frac{\left(L(k)+M^{2}\right) \max }{L(k)+M^{2}},
$$

where $L(k)$ is the lattice Laplacian and $M$ is referred to as the acceleration parameter and is in practice adjusted to the expected value of the correlation length under investigation.

The hermitian conjugate operator of $H$ which we will use below is

$$
H^{\dagger}=\sum_{n m a} \kappa_{n m}\left(D_{n}^{a}+U_{n}^{a}\right) D_{m}^{a} .
$$

We set the common diagonal value of the diffusivity tensor $\kappa_{n n}=K$. The simulation was performed using a two-stage Fourier accelerated RungeKutta algorithm presented in ref [1] in a form appropriate to $O(N)$ groups. For convenience we give the algorithm here in a more general notation. The updating step proceeds by first constructing an intermediate position in configuration space.

$$
S_{n}^{(\mathrm{old})} \rightarrow S_{n}^{(1)}=e^{\epsilon_{n}^{(1) a} \Lambda^{a}} S_{n}^{(\mathrm{old})},
$$

where

$$
\epsilon^{(1) a}=\frac{1}{2} A_{n m} U_{m}^{a} \Delta \tau+\sqrt{\Delta \tau}\left(B^{\frac{1}{2}}\right)_{n m} \eta_{m}^{(1) a} .
$$

The matrices $A$ and $B$ are

$$
A_{n m}=\kappa_{n m}+\Delta \tau C\left[\frac{1}{4}\left(\kappa_{n m}\right)^{2}-\frac{1}{6} K \kappa_{n m}\right],
$$

and

$$
B_{n m}=\kappa_{n m}+\Delta \tau C\left[\frac{1}{4}\left(\kappa_{n m}\right)^{2}-\frac{1}{3} K \kappa_{n m}\right] .
$$

The final step to the updated configuration is

$$
S_{n}^{(\text {old })} \rightarrow S_{n}^{(\text {new })}=e^{\epsilon_{n}^{(2) a} \Lambda^{a}} S_{n}^{(\text {old })},
$$


where

$$
\epsilon^{(2) a}=A_{n m} U_{m}^{(1) a} \Delta \tau+\sqrt{\Delta \tau}\left(B^{\frac{1}{2}}\right)_{n m}\left(\eta_{m}^{(1) a}+\eta_{m}^{(2) a} a\right)
$$

the set of quantities $\left\{\eta_{m}^{(1) a}\right\}$ and $\left\{\eta_{m}^{(2) a}\right\}$ being independent gaussian random numbers with zero mean and unit variance. The superfix (1) on the drift term indicates it has been evaluated at the intermediate configuration $\left\{S_{n}^{(1)}\right\}$.

\section{Variance Reduction}

The method of modifying an estimator so that its mean is unchanged but its variance is reduced has been commonly exploited. In the present case we use the structure of the Langevin process itself to create such variance reduced estimators. The technique is based on the result that the probability distribution $P_{0}(S)$ we use for averaging is the null eigenfunction of the operator $H$ in the Fokker-Planck equation. We have

$$
H P_{r}(S)=E_{r} P_{r}(S)
$$

where $E_{r}<0, \quad r \geq 1$ and $E_{0}=0$. We normalise $P_{0}(S)$ so that

$$
\langle f(S)\rangle=\int d \mu(S) P_{0}(S) f(S)
$$

where $d \mu(S)$ is the group invariant measure on the manifold of spins. Because $H P_{0}(S)=0$ it follows after an appropriate integration by parts that

$$
\langle f(S)\rangle=\int d \mu(S) P_{0}(S) W\left(H^{\dagger}\right) f(S),
$$

where $W(z)$ is any power series in $z$ such that $W(0)=1$. This implies that we can use $f^{\prime}(S)=W\left(H^{\dagger}\right) f(S)$ as an estimator for the same quantity as $f(S)$. There is then the possibility that $f^{\prime}(S)$ has a smaller variance than $f(S)$ itself.

The set $\left\{\psi_{r}(S)=P_{r}(S) / P_{0}(S)\right\}$ are the eigenfunctions of $H^{\dagger}$

$$
H^{\dagger} \psi_{r}(S)=E_{r} \psi_{r}(S)
$$

They satisfy the orthogonality relations

$$
\int d \mu(S) P_{r}(S) \psi_{s}(S)=\delta_{r s} .
$$


Clearly $\psi_{0}(S) \equiv 1$. If we expand $f(S)$ on the set $\left\{\psi_{r}(S)\right\}$ we obtain

$$
f(S)=\langle f(S)\rangle+\sum_{r=1}^{\infty} \alpha_{r} \psi_{r}(S)
$$

for some set of coeficients $\left\{\alpha_{r}\right\}$. The new estimator $f^{\prime}(S)$ is then

$$
f^{\prime}(S)=\langle f(S)\rangle+\sum_{r=1}^{\infty} \alpha_{r} W\left(E_{r}\right) \psi_{r}(S)
$$

and its variance is

$$
\sigma^{2}\left(f^{\prime}\right)=\sum_{r=1}^{\infty}\left(\alpha_{r} W\left(E_{r}\right)\right)^{2} .
$$

When $W(z) \equiv 1, \sigma^{2}\left(f^{\prime}\right)$ reduces to $\sigma^{2}(f)$. If now we minimise $\sigma^{2}\left(f^{\prime}\right)$ with respect to the parameters in $W(z)$ we can expect to find $\sigma^{2}\left(f^{\prime}\right)<\sigma^{2}(f)$. A simple choice which we implement here is $W(z)=1+b z$. Strategies for choosing the value of $b$ are explained in the next section.

\section{Systematic Errors}

The above theory is only applicable to the simulated results on the assumption that the simulated probability distribution has no systematic errors. In fact our Langevin scheme exhibits systematic errors that are $O\left(\Delta \tau^{2}\right)$. We deal with this problem by a statistically controlled extrapolation proceedure combined with variance reduction.

In outline the procedure for evaluating the mean of an observable $f(S)$ is to measure $\langle f\rangle$ and $\langle\hat{f}\rangle$ where $\hat{f}=H^{\dagger} f$ together with the correlators $\left\langle f^{2}\right\rangle,\left\langle\hat{f}^{2}\right\rangle$ and $\langle f \hat{f}\rangle$ for a number of runs with different values of values of $\Delta \tau^{2}$. For the smallest of these values $\Delta \tau_{1}^{2}$ say, we obtain the value of $b$ that yields the minimum variance for the estimator $f^{\prime}=f+b \hat{f}$. We have

$$
\sigma^{2}\left(f^{\prime}\right)=\sigma^{2}(f)+2 b \sigma^{2}(f, \hat{f})+b^{2} \sigma^{2}(\hat{f})
$$

and minimum therefore occurs when

$$
b=-\sigma^{2}(f, \hat{f}) / \sigma^{2}(\hat{f}),
$$

where $\sigma^{2}(f, \hat{f})=\langle f \hat{f}\rangle-\langle f\rangle\langle\hat{f}\rangle$. 
Having established the value of $b$ and hence the precise form of $f^{\prime}$ in this way we compute the $\left\langle f^{\prime}\right\rangle$ and $\sigma^{2}\left(f^{\prime}\right)$ for the other larger choices of $\Delta \tau$. We then do a least squares fit on the assumption that

$$
\left\langle f^{\prime}\right\rangle=\left.\left\langle f^{\prime}\right\rangle\right|_{\Delta \tau=0}+\beta \Delta \tau^{2}
$$

That is we minimise $\chi^{2}$ with respect to $\alpha$ and $\beta$ where

$$
\chi^{2}=\sum_{i} \frac{1}{\sigma_{i}^{2}}\left(\bar{f}_{i}^{\prime}-\alpha-\beta \Delta \tau_{i}^{2}\right)^{2} .
$$

Here $i$ labels the run that uses time step $\Delta \tau_{i}$ and $\bar{f}_{i}^{\prime}$ and $\sigma_{i}^{2}$ are the estimates of the mean and variance of $f^{\prime}$ based on run $i^{\text {th }}$ run. The minimising value of $\alpha$ yields our unbiased estimate for $\langle f\rangle$ and is given by

$$
\alpha=\frac{\left(\sum_{i} \frac{\bar{f}_{i}^{\prime}}{\sigma_{i}^{2}}\right)\left(\sum_{j} \frac{\Delta \tau_{j}^{4}}{\sigma_{j}^{2}}\right)-\left(\sum_{i} \frac{\bar{f}_{i}^{\prime} \Delta \tau_{i}^{2}}{\sigma_{i}^{2}}\right)\left(\sum_{j} \frac{\Delta \tau_{j}^{4}}{\sigma_{j}^{2}}\right)}{\left(\sum_{i} \frac{1}{\sigma_{i}^{2}}\right)\left(\sum_{j} \frac{\Delta \tau_{j}^{4}}{\sigma_{j}^{2}}\right)-\left(\sum_{i} \frac{\Delta \tau_{i}^{2}}{\sigma_{i}^{2}}\right)^{2}} .
$$

The advantage of this proceedure is that it not only provides an estimate of the statistical error on the observable of interest but through the resulting $\chi^{2}$ for the fit provides a test of the assumption of linearity in $\Delta \tau^{2}$ for the systematic error. Our evaluation of the statistical error of our unbiased estimate of $\langle f\rangle$ is $\sqrt{\sigma^{2}(\alpha)}$ where

$$
\sigma^{2}(\alpha)=\sum_{i}\left(\frac{\partial \alpha}{\partial \bar{f}_{i}^{\prime}}\right)^{2} \sigma_{i}^{2}
$$

The detailed treatment of the data involves a systematic re-binning proceedure that we will discuss when we consider decorrelation of measurements in the next section.

The correlation length cannot be measured directly as an observable. It is obtained by fitting simultaneously the estimates for a number of observables, namely the values of the correlation function $G_{t}=\left\langle g_{t}\right\rangle$, where $\left.g_{t}=\operatorname{Tr}\left(\mathcal{S}_{t}^{\dagger} \mathcal{S}_{0}\right)\right\rangle, \mathcal{S}_{t}$ being the spatial average of the spins $\left\{S_{n}\right\}$ for $n$ in time-slice $t$. Our strategy in this case is a generalization of the single operator case discussed above. For a set of values for $\Delta \tau$ we take measurements of the observables $g_{t}$ and the observables $\hat{g}_{t}=H^{\dagger} g_{t}$ forming the correlation matrices $\left\langle g_{t} g_{t^{\prime}}\right\rangle,\left\langle g_{t} \hat{g}_{t^{\prime}}\right\rangle$ and $\left\langle\hat{g}_{t} \hat{g}_{t^{\prime}}\right\rangle$. Our method of proceeding is as 
follows. We construct a set of improved operators $\left\{g_{t}^{\prime}\right\}$ where $g_{t}^{\prime}=g_{t}+b_{t} \hat{g}_{t}$. The coefficients $\left\{b_{t}\right\}$ are to be chosen ultimately to minimise the statistical error on our estimate of the correlation length. For simplicity we worked in fact with a common value $b$ for all the members of the set $\left\{b_{t}\right\}$. It is important to note that the optimal value of $b$ appropriate to the correlation length calculation proved to be different from the value appropriate to the susceptibility calculation.

We then can compute the correlation matrix $\Sigma_{t t^{\prime}}=\left\langle g_{t}^{\prime} g_{t^{\prime}}^{\prime}\right\rangle-\left\langle g_{t}^{\prime}\right\rangle\left\langle g_{t^{\prime}}^{\prime}\right\rangle$ for a given $\Delta \tau$. We make the assumption that

$$
\left\langle g_{t}^{\prime}\right\rangle=\left.\left\langle g_{t}^{\prime}\right\rangle\right|_{\Delta \tau=0}+h_{t} \Delta \tau^{2}
$$

and obtain an unbiased estimate for the Green function $G_{t}=\left\langle g_{t}\right\rangle$ from the extrapolation to $\Delta \tau=0$ by minimising $\chi^{2}$ where

$$
\chi^{2}=\sum_{i t t^{\prime}} \Sigma_{i t t^{\prime}}^{-1}\left(\bar{g}_{i t}-\alpha_{t}-\beta \Delta \tau_{i}^{2}\right)\left(\bar{g}_{i t^{\prime}}-\alpha_{t^{\prime}}-\beta_{t^{\prime}} \Delta \tau_{i}^{2}\right)
$$

where $\bar{g}_{i t}$ is the numerical estimate for $G_{t}$ in the run with $\Delta \tau=\Delta \tau_{i}$, and $\Sigma_{i t t^{\prime}}^{-1}$ is the matrix inverse of $\Sigma_{i t t^{\prime}}$ the corresponding numerical estimate for $\Sigma_{t t^{\prime}}$. The variance matrix for the resulting $\left\{\alpha_{t}\right\}$ is

$$
\sigma^{2}\left(\alpha_{t}, \alpha_{t^{\prime}}\right)=\sum_{i t^{\prime \prime} t^{\prime \prime \prime}} \frac{\partial \alpha_{t}}{\partial \bar{g}_{i t^{\prime \prime}}} \Sigma_{i t^{\prime \prime} t^{\prime \prime \prime}} \frac{\partial \alpha_{t^{\prime}}}{\partial \bar{g}_{i t^{\prime \prime \prime}}}
$$

We use the $\left\{\alpha_{t}\right\}$ as the estimates for the $\left\{G_{t}\right\}$ extrapolated to $\Delta \tau=0$ and the above variance matrix as the estimate for the extrapolated correlator $\left\langle g_{t} g_{t^{\prime}}\right\rangle-\left\langle g_{t}\right\rangle\left\langle g_{t^{\prime}}\right\rangle$. Again we obtain a value for $\chi^{2}$ that measures the goodness of fit for the extrapolation.

To these values we fit the shape

$$
G_{t}=A+B \cosh ((t-L / 2) / \xi),
$$

determining the values of $A, B$ and $\xi$ by minimising

$$
\chi^{2}=\sum_{t t^{\prime}}\left(\alpha_{t}-G_{t}\right) \Sigma_{t t^{\prime}}^{-1}(a)\left(\alpha_{t^{\prime}}-G_{t^{\prime}}\right)
$$

where $\Sigma_{t t^{\prime}}(a)=\sigma^{2}\left(\alpha_{t}, \alpha_{t^{\prime}}\right)$ and $\Sigma_{t t^{\prime}}^{-1}(a)$ is its matrix inverse.

The functional form in eq(30) is not appropriate for lowest few $t$-values where $G(t)$ is appreciably affected by contributions other than the lowest state. We omit these points from the fitting procedure and search for a consistent fit at the higher values of $t$ with an acceptable chi ${ }^{2}$ of about unity per degree of freedom. 


\section{Singular Value Decomposition of $\Sigma$}

Because $\Sigma_{t t^{\prime}}$ has some very small eigenvalues it is possible that there can be some difficulty in inverting this matrix and hence in obtaining a meaningful evaluation of $\mathrm{chi}^{2}$. However, this problem can be circumvented by exploiting the singular value decomposition (SVD) of $\Sigma[7]$ and following the procedure suggested by Lepage in ref [8].

The data for $G_{t}$ for different values of t are highly positively correlated. This means that the estimates for $G_{t}$ lie on a smoother curve than the individual errors on each point would suggest. It also means inevitably that there are eigenvectors of $\Sigma$ associated with very small eigenvalues and that these eigenvectors have entries which change in sign: a behaviour which is unphysical and which is not represented in the fitting function eq(30). For a large lattice there are many such "unphysical" eigenvectors and their associated amplitudes in the expansion of $G_{t}$ on the eigenbasis each contribute to $\mathrm{chi}^{2}$ weighted by the inverse of a small eigenvalue i.e., a large number. We then note that the eigenvalues are only statistical estimates for the true variances, that the number of unphysical parameters grows with lattice temporal size and that their contribution to $\mathrm{chi}^{2}$ is strongly weighted compared with those of the more physical eigenvectors.

The naive fitting procedure can then produce unsatisfactory results since chi $^{2}$ is dominated by unphysical modes and a moderate change in the fitting parameters has very little effect on their contribution to $\mathrm{chi}^{2}$ since the smooth fitting function has little overlap with the unphysical modes. The consequence is that large variations in the parameters occur in an attempt to reduce the bulk contribution to $\mathrm{chi}^{2}$ and the fitted values are thus controlled not by the physical signal but by statistical fluctuations in amplitudes known to be zero. This can result in a fitted function which clearly does not pass through the smooth set of data points anywhere near as well as it should. To remedy the situation the inverse of $\Sigma$ is computed by first making a singular value decomposition and then inverting the eigenvalues but replacing the inverse of all but the largest ones by zero. This removes the effect of unphysical modes and means that we need not add oscillatory fitting functions whose sole role is to soak up the statistical fluctuations on amplitudes known to be zero. Note that we include the constant function as it has an appreciable expansion on modes of $\Sigma$ with large eigenvalues.

In our fitting procedure we keep about six modes but check the the results are stable for all reasonable choices. 


\section{$6 \quad$ Numerical Results}

Our numerical results were obtained at a couplings $g=1.5$ on the $32 \times 32$ and $g=1.75$ on the $64 \times 64$ lattices respectively. Measurements were taken every two updates after equilibriation at values $\Delta \tau^{2}=0.04,0.06 \& 0.08$ with a squared fourier acceleration mass of $M^{2}=.02$. The total number of iterations used was $48 \times 10^{3}$, with $32 \times 10^{3}$ at $\Delta \tau^{2}=0.04$, and $8 \times 10^{3}$ at both of $\Delta \tau^{2}=0.06 \& 0.08$. We employed a higher number of iterations at the lowest value of $\Delta \tau^{2}$ because of its importance in the extrapolation procedure to $\Delta \tau^{2}=0$.

The results are accumulated according to a standard re-binning procedure in which means, mean squares and cross correlators are compiled from individual results then from the averages of pairs, quadruples etc. By computing the variance of an observable from a sequence of bins and noting the point at which the it stabilises we can estimate a value for the Langevin correlation time of the observables in question. The value we quote $\tau_{\chi}$, is that appropriate to the susceptibility. By applying this approach with and without operator improvement we can detect the effect of operator improvement on the Langevin decorrelation time.

In Table 1 we exhibit the results of our procedure for the susceptibility $\chi$, and the Langevin decorrelation time $\tau_{\chi}$ for lattice sizes of $32 \times 32$ and $64 \times 64$. In Table 2 we show the results for the correlation length $\xi$. The values of $\mathrm{chi}^{2}$ per degree of freedom are for the $\Delta \tau^{2}$-extrapolation. The results for $\chi$ and $\xi$ compare quite well with the corresponding results obtained by Meyer who used a multi-grid method. We have comparable or smaller statistical errors for a smaller number of iterations of the update.

Fig 1 shows a comparison between extrapolations for the susceptibility on the $64 \times 64$-lattice with and without the operator improvement. The value of $\mathrm{chi}^{2}$ for these extrapolations were very much less than unity indicating the assumption of $O\left(\Delta \tau^{2}\right)$ for the systematic errors was well verified. It is clear from Fig. 1 that the operator improvement also leads to a reduction in the the systematic error, a fact which helps to improve the extrapolation procedure by reducing the obliqueness of the angle at which the extrapolation line hits the $\Delta \tau^{2}=0$ axis. The reason may be inferred from the following analysis. If we accept that our choice for $W(z)=1+b z$ is an approximation to the choice $W(z)=\exp \{b z\}$ then we see that our operator improvement is an approximation to $f^{\prime}(S)=\exp \left\{b H^{\dagger} f(S)\right\}$. The simulation results in a probability distribution $P_{\operatorname{sim}}(S)$ which is in error by 
$O\left(\Delta \tau^{2}\right)$. The simulation average of the improved operator is then

$$
\left\langle f^{\prime}(S)\right\rangle_{\operatorname{sim}}=\int d \mu(S) P_{\operatorname{sim}}(S) e^{b H^{\dagger}} f(S)
$$

Integration by parts yields

$$
\left\langle f^{\prime}(S)\right\rangle_{\operatorname{sim}}=\int d \mu(S)\left(e^{b H} P_{\operatorname{sim}}(S)\right) f(S) .
$$

The effective probability distribution is therefore $P_{\text {eff }}(S)=\exp \{b H\} P_{\text {sim }}(S)$. Clearly $P_{\text {eff }}(S) \rightarrow P_{0}(S)$ as $b \rightarrow \infty$. Our variance reduction method does yield positive values of $b$ because the operator improvement correlates strongly and negatively with the original estimator $(\mathrm{eq}(22))$. That this must be the case follows directly from the non-positive nature of $H^{\dagger}$. Consequently, it is plausible that we can also expect a reduction in the systematic error for the improved operator. We find that this is indeed the case. The negative correlation is illustrated in Fig. 2 where we have plotted the improved operator results for the various values of $\Delta \tau^{2}$ against $b$. The bands represent the the result \pm the statistical error and not only exhibit the expected narrowing corresponding to minimum variance but also show a tendency to converge around a region encompassing the true result thus indicating that the operator improvement compensates in a consistent way for the systematic error. This latter compensation is not perfect of course but is not required to be and might be further improved by including additional $\left(H^{\dagger}\right)^{2}$ terms in the operator improvement.

In Figs. $3 \& 4$ we show the extrapolated correlation function $G_{t}$ together with the fit obtained by the above procedure for $32 \times 32$ and $64 \times 64$ lattices. In line with our general procedure these fits were obtained with the omission of the first three $t$-values. The values for the $\mathrm{chi}^{2}$ per degree of freedom were approximately unity. The ommission of further $t$-values changed the fitted parameters only by small amounts well within the statistical errors.

From the results in Table 1 it is clear that operator improvement drastically reduces the Langevin correlation time. In the case of the $32 \times 32$-lattice the reduction is by a factor of 3.5 from 14 time-steps down to 4 and for the $64 \times 64$-lattice by a factor of 4 from 16 to 4 . These speeded up decorrelations can be plausibly interpreted as the origin of the reduced variances that we achieved after operator improvement. A comparison of our results with those of ref [3] suggests that for a given number of iterations our statistical errors are better by a factor of a little more than two. Translated into numbers of iterations this becomes a factor of approximately four or five. 
Allowing for differences in computers we estimate that our CPU update time is approximately six times greater than that of refs [3, 价. The net result is that we achieve, for $N=3$ on the sizes of lattice we have so far been able to deal with, a given error in a comparable real time notwithstanding our need to accumulate data at different values of $\Delta \tau$ in order to perform the extrapolation needed to eliminate the systematic error.

A comparison of methods needs a detailed numerical study. Some general points can be made however. The time consuming parts of the update step that we use are the exponentiation of the generators to compute $S U(3)$ group elements and the Fourier transform procedure required for the implementation of the Fourier accelerated update. The exponentiation of the generators to produce finite group elements is at the centre of the various updating methods. Hasenbusch and Meyer [3, 国 simplify this problem by organising the algorithm so that only diagonal generators need to be exponentiated. This leads to a dependence on $N$ for their algorithm that is $O(N)$. In our case for $S U(N) \times S U(N) / S U(N)$ the dependence is necessarily $O\left(N^{3}\right)$. For $C P(N)$, which we have not investigated in this paper, it would be $O\left(N^{2}\right)$. Superficially then it would seem that our Langevin scheme would lose rapidly to the updating technique used by Hasenbusch and Meyer for larger $N$ values. However the outcome of any comparison depends also on the efficacy of the update in covering the configuration space of the spins. We have seen that per update our method augmented with operator improvement does a relatively better job in producing independent configurations. There are grounds to believe that this relative advantage will increase with $N$ and that the method will remain competitive with that of Hasenbusch and Meyer [3, 那. Only a numerical trial however can properly settle this issue.

We are presently applying the Langevin method with operator improvement to larger lattices in order to test for the occurence of critical slowing down. A vitally important aspect of the problem that we have not fully addressed in this paper.

\section{Acknowledgements}

RRH would like to thank the Theory Division CERN for their hospitality during a sabbatical year when much of this work was carried out, and he would also like to thank U. Wolff for useful discussions. 


\section{Tables}

Table 1

\begin{tabular}{|l|l|l|c|c|r|}
\hline$g$ & $L$ & $b_{\chi}$ & $\chi$ & $\mathrm{chi}^{2}$ & $\tau_{\chi}$ \\
\hline \hline 1.5 & 32 & 0.0 & $59.0(7)$ & 0.02 & 14 \\
\hline 1.5 & 32 & 0.6 & $57.7(4)$ & 0.52 & 4 \\
\hline 1.75 & 64 & 0.0 & $298.8(4.2)$ & 0.76 & 11 \\
\hline 1.75 & 64 & 0.8 & $301.5(2.8)$ & 0.003 & 3 \\
\hline
\end{tabular}

\section{Table 2}

\begin{tabular}{|l|l|l|c|r|}
\hline$g$ & $L$ & $b_{\xi}$ & $\xi$ & chi $^{2}$ \\
\hline \hline 1.5 & 32 & 0.0 & $3.04(3)$ & 0.94 \\
\hline 1.5 & 32 & 0.2 & $3.05(2)$ & 1.2 \\
\hline 1.75 & 64 & 0.0 & $8.39(09)$ & 1.5 \\
\hline 1.75 & 64 & 0.2 & $8.41(07)$ & 1.5 \\
\hline
\end{tabular}

\section{Table Captions}

Table 1. List of results for the susceptibility $(\chi)$ on two lattices with and without operator improvment. The $\mathrm{chi}^{2}$ per degree of freedom is shown for the $\Delta \tau^{2}$-fit.

Table 2. List of results for the correlation length $(\xi)$ on two lattices with and without operator improvment. The chi ${ }^{2}$ per degree of freedom is shown for the $\Delta \tau^{2}$-fit.

\section{Figure Captions}

Fig. 1. Plot of the susceptibility against $\Delta \tau^{2}$ on a $64 \times 64$-lattice. The upper and lower curves show the results with and without operator improvement.

Fig. 2. Plot of susceptibility on a $64 \times 64$-lattice against the improvement parameter $b$ for $\Delta \tau^{2}=0.04$ (continuous line), 0.06 (light 
dashes) \& 0.08 (heavy dashes). The bands indicate the statistical error.

Fig. 3. The correlation function on a $32 \times 32$-lattice for coupling $g=1.5$.

Fig. 4. The correlation function on a $64 \times 64$-lattice for coupling $g=1.75$. 


\section{References}

[1] S M Catterall, I T Drummond and R R Horgan, Phys. Letts. B254, $177,(1991)$

[2] U Wolff, Phys. Rev. Lett. 62361 (1989) and Nucl. Phys. B334 581 (1990)

[3] M Hasenbusch S Meyer, Phys. Rev. Lett. 68435 (1992)

[4] M Hasenbusch and S Meyer, Phys. Rev. D45 R4376 (1992)

[5] R G Edwards, S J Ferreira, J Goodman and A D Sokal, Nucl. Phys. B380 621 (1992)

[6] I T Drummond and R R Horgan, Nucl. Phys. B (Proc. Suppl.) 26629 (1992)

[7] W H Press, B P Flannery, S A Teukolsky, W T Vetterling, 1986, Numerical Recipes, Cambridge University Press.

[8] B A Thacker and G P Lepage, Phy Rev D, 43, 196 (1991)

[9] I T Drummond, S Duane and R R Horgan, Nucl. Phys. B220 119 (1983)

[10] G G Batrouni et al, Phys. Rev. D 322735 (1989) 